\title{
Stimulus and response meaningfulness in paired-associate learning'
}

\author{
LOLA L, CUODY, QUEEN'S UNIVERSITY, KINGSTON, ONTARIO, CANADA \\ TANMIS Y. ARBUCKLE, SIR GEORGE WILLIAMS UNIVERSITY, MONTREAL, QUEBEC, CANADA
}

The effects of stimulus and response meaningfulness (m) on paired-associate leaming and retention were examined under conditions where stimuli and responses were made equally available at the time of testing by means of a matching technique. Two combinations of stimulus and response meaningfulness were compared: high meaningful stimulus and low meaningful response (H-L), and low meaningful stimulus and high meaningful response $(\mathrm{L}-\mathrm{H})$. No differences were found between the number of $\mathrm{L}-\mathrm{H}$ pairs and the number of $\mathrm{H}-\mathrm{L}$ pairs correctly matched. A significant effect of rated pair difficulty was found.

When stimulus and response members of a paired associate differ in meaningfulness $(m)$, the combination of a low meaningful (L) stimulus with a high meaningful (H) response ( $\mathrm{L}-\mathrm{H})$ has been found to produce more rapid learning than does the reverse (H-L) combination. Underwood \& Schulz (1960) interpret this finding as being due to the avallability of the response term; the $\mathrm{H}$ responses are more avallable than the $L$ responses. Where $H$ and $L$ responses are made equally available, $L-H$ and H-L pairs should be equally easily learned. But when Martin, Cox, \& Boersma (1965) attempted to control for response availability by using a multiple-choice recognition technique in which all eight responses of a list were presented with each stimulus item, they found that the effect of response $m$ was greater than that of stimulus $\underline{m}$; thus $L-H$ pairs were better recalled than $H-L$ pairs.

Certain methodological issues need to be resolved before the Martin etal results can be used to challenge the Underwood and Schulz explanation. One issue is whether the limitation of recall time to 4 sec.per stimulus item unculy favored the $H$ responses, as Lovelace \& Schulz (1966) have suggested. It may take less time to locate an $H$ response than to locate an $L$ response. A second issue is whether the presentation of all responses with each stimulus, which might be assumed to increase intratrial interference, could have resulted in differential forgetting of the H-L and L-H associations.

The present experiments tested for differences in recall between $\mathrm{H}-\mathrm{L}$ and $\mathrm{L}-\mathrm{H}$ order of pairs under conditions where (a) response availability was controlled by means of a matching technique, (b) location-time was not a factor, and (c) the possibility of differential forgetting of $\mathrm{H}-\mathrm{L}$ and $\mathrm{L}-\mathrm{H}$ pairs could be evaluated by employing two recall tests (RTT paradigm).

Methed

The Ss were 144 male inmates of the Kingston Peniten- tiary, who had a median age of 28 years, a median IQ of 102, and a median educational level of elghth grade.

Four sets of six pairs were constructed according to the rules of Noble \& McNeely (1957) using items from the $H$ and L extremes of Noble's (1952) $\mathrm{m}$ scale. Each pair consisted of one $H$ item and one $L$ item. Sets 1 and 2 were unstandardized as to pair difficulty; Sets $3 \mathrm{E}$ and $4 \mathrm{D}$ consisted of pairs which had previously been rated easy (E) and difficult (D), respectively, by 40 students of Queen's University. The pairs for Set 3E and 4D were chosen so that the mean $m$ values of the two sets were nearly equal (4.5 for Set 3E, 4.7 for Set 4D). All pairs were printed separately on $3 \times 5$ in. file cards, once in the L-H order and once in the H-L order, thus yielding eight different six-pair lists (four sets by two orders).

Two different recall sheets, one for each recall test ( $T_{1}$ and $T_{2}$ ) were constructed for each of the eight lists. The six stimulus items were printed in a random order down the left side of the sheet; the corresponding six response items were printed in a different random order down the right side of the sheet (with the restriction that no pair of items be thus matched) and lines were drawn beside each stimulus member down the middle of the sheet.

Two experiments were conducted. Within each experiment, 18 Ss were randomly assigned to one of four groups, each group learning two lists as outlined in Table 1.

All Ss were tested individually and were given preliminary practice recall of three pairs of items unrelated to the experimental materials. In the experiments, pairs were presented manually at a 2-sec. rate in one of six different sequences (cf., Tulving \& Arbuckle, 1963). Each sequence was administered to three of the $18 \mathrm{Ss}$ in a group. Following presentation of the first list, $S$ was given the appropriate $T_{1}$ recall sheet and was in-

Table 1. Outline of conditions in Experiments 1 and 2

\begin{tabular}{lcll} 
Experiment & Group & First List & Second List \\
\hline 1 & 1 & Set 1 H-L & Set 2 L-H \\
& 2 & Set 1 L-H & Set 2 H-L \\
& 3 & Set 2 H-L & Set 1 L-H \\
& 4 & Set 2 L-H & Set 1 H-L \\
2 & 1 & Set 3E H-L & Set 4D L-H \\
& 2 & Set 3E L-H & Set 4D H-L \\
& 3 & Set 4D H-L & Set 3E L-H \\
& 4 & Set 4D L-H & Set 3E H-L \\
\hline
\end{tabular}


structed to fill in the blanks with the correct response term for each stimulus term, guessing where he was not sure. After he had completed recall (Experiment 1), or after 60 sec. had elapsed (Experiment 2), he was given the appropriate $T_{2}$ recall sheet and told to proceed as on $T_{1}$. The second list was then presented, and the two recall tests conducted as with the first list. Resulis

Mean number of correct matches per list averaged across all conditions were as follows: Experiment 1: $T_{1}=2.9, T_{2}=1.9 ;$ Experiment 2: $T_{1}=3.2, T_{2}=2.9$. Separate analyses of variance were performed on these four sets of data. In no analysis was any significant difference found between recall of $L-H$ and $H-L$ pairs, nor did this variable interact either with stage or practice (first vs, second list learned) or with list-set. In Experiment 1, the mean number of correct matches for the $L-H$ pairs was 2.8 and 1.8 for $T_{1}$ and $T_{2}$, respectively, and for the H-L pairs, 3.0 and 2.1. Corresponding values for Experiment 2 were: $L-H$ pairs, 3.2 and 2.9, H-L pairs, 3.2 and 2.8. The one significant effect in Experiment 1 occurred in the analysis of $T_{2}$ recall, where the second list learned was retained better than the first list learned. Mean number of correct matches for the first list was 1.7; for the second list, 2.14 ( $F=$ 4.7, $\mathrm{df}=1 / 68, \mathrm{p}<.05)$. This effect, however, was not replicated in Experiment 2. In Experiment 2, Set $3 \mathrm{E}$ was recalled significantly better than was Set 4D on both $T_{1}$ and $T_{2}$. The mean number of correct matches for $T_{1}$ was: Set $3 E=4.07$, Set $4 D=2.36(F=47.2$, $\mathrm{df}=1 / 68$, $p<.001$ ), and for $T_{2}$ was: Set $3 E=3.67$, Set $4 D=2.04$ ( $F=32.8, \mathrm{df}=1 / 68, \mathrm{p}<.001)$.

In Experiment 1, Ss took a mean of $96 \mathrm{sec}$. to complete $\mathrm{T}_{1}$ matches and a mean of $99 \mathrm{sec}$. to complete $\mathrm{T}_{2}$ matches. In Experiment 2, where recall time was restricted to $60 \mathrm{sec}$, per test, nearly half the Ss omitted one or more matches on $T_{1}$ or $T_{2}$. Analysis of the omissions, however, did not reveal any significant differences between $\mathrm{L}-\mathrm{H}$ and $\mathrm{H}-\mathrm{L}$ pairs. Both on $\mathrm{T}_{1}$ and on $\mathrm{T}_{2}$ approximately $50 \%$ of the Ss who omitted one or more matches made more omissions with the $\mathrm{L}-\mathrm{H}$ pairs while the remaining $50 \%$ made more omissions with the $\mathrm{H}-\mathrm{L}$ pairs. Analysis of the differences in omissions for the pairs rated difficult (Set 4D) and for the pairs rated easy (Set $3 E$ ), however, indicated that there were significantly more omissions in recall of set $4 D$ than in recall of Set $3 E$ for both $T_{1}$ and $T_{2}$. On $T_{1}$, there were $24 \mathrm{Ss}$ making more omissions on Set $4 \mathrm{D}$ than on Set $3 E$ while only five Ss showed the reverse; on $T_{2} 29$
Ss showed more omissions on Set 4D than on Set 3E, with two Ss showing the reverse (for $T_{1}, z=3.3, p<.001$; for $T_{2}, z=4.8, p<.001$, sign test for correlated samples).

Discussion

The findings of these experiments support the Underwood \& Schulz (1960) hypothesis that when the response terms are equally available $\mathrm{H}-\mathrm{L}$ and $\mathrm{L}-\mathrm{H}$ pairs will be learned with equal facility. The present finding that $H-L$ pairs are as well recalled on $T_{2}$ as $L-H$ pairs indicates that there is no difference in rate of forgetting of associations as a function of their direction. Finally, the fact that pairs in a $\mathrm{H}-\mathrm{L}$ or $\mathrm{L}-\mathrm{H}$ order are recalled equally well, independent of the effect of rated difficulty of the pairs, suggests that in general the associative phase of PA learning is not affected by the order in which the two terms to be associated are presented.

It seems quite possible that the differential effects of stimulus $\underline{m}$ and response $\underline{m}$ found by Martin et al are due to the severe restriction on time for scanning and locating response items. In the present experiments, recall time was greatly increased so that $S$ was allowed time to read all response items. The omissions in Experiment 2 most likely reflect an inability of Ss to make decisions within the allotted time despite instructions to guess. One might now investigate the apparent relations between decision-making and rated item difficulty.

\section{References}

Lovelace, E. A., \& Schulz, R. W. Comments on Martin, Cox and Boersma. Psychon. Sci., 1966, 5, 72.

Martin, C. J., Cox, D. L., \& Boersma, F. J. The role of associative strategies in the acquisition of p-a material: an alternative approach to meaningfulness. Psychon, Sci., 1965, 3, 463-464.

Noble, C. E. An analysis of meaning. Psychol. Rev., 1952, 59, 421-430.

Noble, C. E., \& McNeely, D. The role of meaningfulness $(m)$ in paired-associate verbal learning. J. exp. Psychol., 1957, 53, 1622.

Tulving, E., \& Arbuckle, T. Y. Sources of intratrial interference in immediate recall of paired associates. J. verbal Learn. verbal Behav., 1963, 1, 321-334.

Underwood, B. J., \& Schulz, R. W. Meaningfulness and verbal learning. Philadelphia: Lippincott, 1960.

\section{Note}

1. This research was supported by a grant to L. Cuddy from Queen's Committee for Scientific Research. The authors thank Dr. George Scott, Director of Psychiatric Services, Kingston Penitentiary, for making the subjects available to them, and Mr. P. Gendreau for collecting and analysing the data. 